\title{
Criminologie
}

\section{La revue Criminologie et la recherche}

\section{Christian Debuyst}

Volume 10, numéro 2, 1977

La criminologie au Québec

URI : https://id.erudit.org/iderudit/017075ar

DOI : https://doi.org/10.7202/017075ar

Aller au sommaire du numéro

Éditeur(s)

Les Presses de l'Université de Montréal

ISSN

0316-0041 (imprimé)

1492-1367 (numérique)

Découvrir la revue

Citer cet article

Debuyst, C. (1977). La revue Criminologie et la recherche. Criminologie, 10(2), 64-77. https://doi.org/10.7202/017075ar d'utilisation que vous pouvez consulter en ligne.

https://apropos.erudit.org/fr/usagers/politique-dutilisation/ 
Le dixième anniversaire de la revue Acta Criminologica qui, depuis deux ans, a pris cette nouvelle forme que nous connaissons, est l'occasion, non pas tellement de rappeler le travail accompli au cours de cette période déjà longue, mais de rechercher à travers les nombreuses études publiées les points à partir desquels une discussion peut être engagée et poursuivie. $\mathrm{La}$ fécondité d'une revue, en effet, réside dans le fait que sa lecture, ou sa relecture, puisse rendre de tels débats possibles, et que la preuve soit ainsi faite de ce qu'elle n'a cessé de s'inscrire dans les préoccupations que l'actualité suscite.

Du fait même du développement considérable de la criminologie au Québec, la nouvelle revue, Criminologie, qui constitue le prolongement des Acta, est plus directement centrée sur les préoccupations du Québec dans les multiples domaines qui entrent dans le cadre des sciences criminologiques. C'est la raison pour laquelle cette chronique des activités passées a surtout porté sur les contributions contenues dans les Acta qui émanent des membres du département de criminologie et plus tard, des différents centres nés du développement de ce dernier.

Que l'auteur de cette chronique soit un membre extérieur au cadre québécois peut être utile, car l'histoire de la criminologie, avec ses fluctuations, se révèle d'une manière presque exemplaire à travers cette production d'une dizaine d'années, et dès lors, un regard extérieur peut donner lieu à une vue plus dégagée. Mais cependant, en ayant lui-même à de nombreuses reprises participé aux activités du département, ce regard extérieur n'est pas un regard étranger. Il en résulte que cette chronique deviendra forcément, à certains endroits, une chronique partiale, parce qu'elle constitue le prolongement de discussions antérieures et qu'elle ne peut d'aucune manière être considérée comme s neutre s. C'est là, peuton dire, la manifestation même d'un attachement.

Nous avons partagé cette chronique en deux parties : la première vise à décrire l'implantation, depuis la création du département, d'une politique et d'une pratique criminologiques dans le cadre québécois, avec les points d'interrogation qu'elle suscite. Dans la deuxième, nous essayerons de présenter et de discuter les apports des articles publiés dans les Acta à la problématique criminologique telle qu'elle s'est développée au cours de ces dernières années. Ce n'est pas une tâche facile, étant donné la multiplicité de ces apports. Un tel essai nous a cependant paru justifié.

La création du département de criminologie dans le cadre de la faculté des sciences sociales (1960) a été, pour le professeur Denis Szabo, un véritable pari : un pari qui se situe d'abord au niveau théorique et qui l'amène à opter pour une criminologie considérée comme science autonome, ayant sa spécificité, ayant ses méthodes de recherche, son approche clinique propre; pari qui se situe également au niveau d'une politique concrète : celle de l'intégration d'une nouvelle profession dans le contexte social. Sans doute les conditions étaient-elles favorables; mais il faut reconnaître que rarement, on aura pu constater cet investissement systématique d'un secteur, qui s'est traduit à la fois au niveau du développement 
des idées et à celui de l'occupation du terrain où le professionnel criminologue pouvait prendre place et remplir son rôle propre.

Nous n'allons pas aborder le débat théorique qui pourrait être soulevé à propos de la criminologie comme science autonome. Cette question s'est modifiée considérablement au cours des dix dernières années. En plus, dans les différentes publications, elle n'a jamais été posée explicitement en ces termes. La voie suivie fut plutôt celle qui consista à prouver le mouvement en marchant, c'est-à-dire à prouver que la criminologie était une science et une pratique en exerçant cette science et cette pratique, et en provoquant une reconnaissance de cette démarche particulière dans le milieu social ambiant. Nous le verrons clairement dans deux domaines différents : celui d'abord de la politique générale face au crime, celui ensuite de la clinique criminologique.

La période que nous pourrions appeler héroïque fut sans doute celle au cours de laquelle il fallut prouver aux instances responsables qu'une étude systématique du phénomène délinquant et de la réaction sociale constituait l'élément indispensable à partir duquel une politique pouvait être pensée, et que dans le cadre d'une telle préoccupation, un département de criminologie à l'université était une pièce essentielle.

A première vue, cette perspective peut paraître évidente. Mais ce qui paraîtra tel au niveau des idées ne l'est pas nécessairement au niveau de la réalité pratique où les facteurs déterminants sont généralement les habitudes acquises, les intérêts des différents groupes en présence, les réactions émotionnelles de défense, etc.

Dans le contexte québécois, il faut reconnaître qu'une inscription de la perspective criminologique dans la politique fut grandement facilitée par l'existence d'une pratique, inconnue dans les pays de l'Europe continentale : celle qui consiste à constituer des commissions d'enquêtes destinées à faire le point sur les questions critiques de l'heure, et dans lesquelles des personnes compétentes, n'ayant pas de mandat politique particulier, sont à même de donner un avis. C'est, directement ou indirectement, en relation avec une telle pratique qu'une série de grandes enquêtes ont été menées, sous la direction de D. Szabo, directeur du département, par ses collaborateurs, que ce soient les enquêtes d'opinion publique sur la police au Québec (Rico, J.M., Tardif, G., 1969), celle sur la justice criminelle (Fattah, E., Normandeau, A., 1969), ou encore dans le domaine des mineurs, les études comparatives sur les tribunaux pour mineurs (Parizeau, A., 1969). Une telle activité s'est prolongée par la suite, selon d'autres modalités (commission fédérale sur les drogues, comité d'étude sur la réadaptation des enfants et adolescents placés au centre d'accueil); mais cela semble bien être par ce canal qu'une nouvelle forme de raisonnement et de préoccupations, liées aux sciences criminologiques, a pris place dans des secteurs qui généralement y sont étrangers ou même hostiles. C'est d'ailleurs dans le même mouvement mais selon d'autres formules que, plus tard, le C.I.C.C. élargira ses préoccupations au niveau international, et plus particulièrement à celui des pays du tiers monde (voir les séminaires organisés par le C.I.C.C. dans ces pays et les nombreux rapports qui en ont résulté).

Un tel investissement - que ce soit dans l'un ou l'autre de ces secteurs - n'est évidemment pas sans soulever des problèmes, du fait qu'un même type d'approche peut, avec le temps, changer de signification ou prendre une signification ambiguë, qui varie selon le point de vue de 
ceux qui l'apprécient. Dans un premier temps, en effet, l'apport de la criminologie au droit et aux pratiques liées à son application, se situe dans une perspective nettement novatrice et oblige les institutions à prendre en considération des éléments nouveaux et méconnus. Mais après ce premier temps, un tel apport, même s'il maintient son caractère novateur, n'apparaît effectivement que comme un a complément * qui rend une attitude de départ plus nuancée, plus complexe, mais qui ne la transforme pas, ou la transforme d'autant moins que celle-ci apparaît comme liée et dépendante des structures sociales en place. La criminologie, dans cette optique et même à travers les aspects positifs qui la caractérisent, apparaît comme un instrument au service du pouvoir. Il en résulte une ambiguité selon ce qu'on pense de ce pouvoir. Même s'il existe un accord sur le caractère novateur que doit avoir lapport criminologique, la question se posera : novateur par rapport à quoi et par rapport à qui ? Ce qui caractérise, nous semble-t-il, la situation québécoise, c'est que, du fait même des habitudes déjà prises, le débat s'est situé plus nettement dans le cadre d'une politique concrète et tend à s'exprimer par des prises de position face à des questions précises, avec toutes les nuances qui en résultent. Nous connaissons en effet peu de pays, et même nous n'en connaissons aucun, où les criminologues soient aussi présents dans les débats publics qui touchent à la délinquance ou, plus largement, à la déviance sociale; peu de pays aussi où ils soient plus soucieux de justifier leur pratique.

A côté de cette intrusion đu criminologue dans les affaires publiques, nous avons un autre problème majeur : celui du développement de la profession de criminologue dans le cadre de la clinique, on pour utiliser le terme qui avait cours au départ, dans le cadre de la resocialisation. Là également, dès la création du département, comme nous l'avons dit, un pari a été fait : celui de créer, pour le criminologue, un champ professionnel suffisamment repérable pour que, parmi les autres professions, il trouve sa place sans ambiguïté.

Le Québec présentait à l'époque une situation favorable, en ce sens que les activités parajudiciaires (particulièrement celles d'agent de probation, pour les adultes comme pour les mineurs) n'étaient pas déjà occupées par d'autres professions (assistants sociaux, etc.) comme c'est généralement le cas dans la plupart des pays européens. Il en résulte un développement relativement rapide de la fonction dans les services judiciaires, pénitentiaires ou administratifs. Néanmoins, l'important ne se situe pas à ce niveau : il ne s'agit pas seulement de l'occupation d'un champ professionnel resté vide, il s'agit aussi, en premier lieu, de donner à ce professionnel un outil de travail adéquat, et en second lieu, d'élargir son intervention, particulièrement dans certains secteurs comme celui de la jeunesse, à des champs extrajudiciaires. Ces deux points valent la peine d'un examen plus approfondi, car ce sera sur ces deux questions que des perspectives comme d'ailleurs des interrogations nouvelles pourront se dégager.

La première question est celle de l'outil qu'il importe de fournir au criminologue pour qu'il puisse travailler d'une manière adkquate. Si l'on veut résumer l'évolution des efforts menés dans ce domaine, on peut dire, me semble-t-il, qu'une nécessité est apparue : celle de donner au criminologue une compétence pour aborder le comportement socialement inadapté. On constate en effet que cet outil a d'abord été défini comme outi] de diagnostic, ou comme instrument d'évaluation de la personnalité. Le problème consistait à donner au criminologue-clinicien une possibilité d'éva- 
luation suffisamment sérieuse pour que, dans la majorité des cas auxquels il se trouve confronté, il puisse valablement apprécier les a ressources , individuelles dans le cadre qui lui importe particulièrement, celui des interrelations, et qu'il puisse, à partir de là, élaborer un programme d'action. C'est à ce niveau qu'un effort a été mené, et continue d'être mené, tout particulièrement par $\mathbf{M}$. Fréchette et ses collègues cliniciens et méthodologues. De la même manière et dans la même perspective, il importait également de donner au criminologue-clinicien les outils lui permettant de préciser les différents soutiens que dans le milieu familial et social, il lui était possible de trouver pour établir et réaliser son plan d'action. Les instruments présentés par Marineau (1974) ainsi que les efforts de rationalisation menés dans ce domaine tant au niveau de la prévention que du traitement constituent des * appuis " de plus en plus solides.

Sa fonction étant conçue en ces termes et pour autant que ces deux moments de sa formation soient menés à bien, le criminologue-clinicien avait ou devenait susceptible d'acquérir les qualités requises pour jouer ce rôle de enégociateur s entre celui dont la conduite révèle des difficultés d'intégration et d'autre part le milieu qui pose un problème d'exigences à ceux qui le constituent. C'est dans ce sens, me semble-t-il, que le criminologue cumule certaines fonctions du psychologue et certaines fonctions de l'assistant social, en leur donnant néanmoins une originalité, celle d'établir un lien entre ces deux fonctions dans un secteur où, généralement, ce lien n'existe pas ou est mal défini.

Cette description est sans doute une description idéale et nous sommes très conscient que dans un grand nombre de cas, le criminologue clinicien n'a pas la possibilité de mener à bien cette double formation (d'où cette impression d'être assis entre deux chaises), ou encore, que les conditions concrètes dans lesquelles il travaille ne lui donnent pas la possibilité d'exercer cette tâche complexe. Ce qui néanmoins reste vrai, est que tel paraît bien avoir été le projet de départ, même s'il n'a pas été explicitement formulé, et qu'en plus, dans certaines circonstances, un tel projet se réalise effectivement (nous rappelons ici à titre d'exemple l'équipe de M. Côté au C.S.S. de Longueuil).

Si une telle question nous paraît essentielle, ce n'est pas seulement parce qu'il importe de préciser l'outil du criminologue et la manière dont il l'exercera. C'est aussi parce que, à travers l'analyse de cette fonction particulière, on pose le premier jalon d'un problème plus large qui est celui d'une redéfinition des différentes fonctions existant dans le cadre des sciences humaines. Sous la pression des lois et sous celle d'une fausse rationalité, on tend en effet à vouloir fragmenter les tâches et à les partager entre les différents professionnels qui ne seraient compétents que pour des segments d'intervention (les uns pour poser le diagnostic, les autres pour décider de la mesure à prendre, les troisièmes pour mettre la mesure à exécution, etc.). Nous touchons là une question cruciale qui, avec les projets de modification de la loi sur la protection de la jeunesse, s'affirmera d'une manière de plus en plus aiguë (v. le $\mathrm{n}^{0}$ de 1975 de la revue Criminologie). Il nous est malheureusement impossible de nous y attarder plus longuement.

Le deuxième point qu'il importe de traiter dans cette analyse de la profession de criminologue-clinicien est celui de l'élargissement de ses possibilités d'intervention. Dans le cadre des activités du département, un tel développement s'est fait particulièrement dans le domaine juvénile où étaient possibles, et même souhaitables, le repérage de ceux qui furent 
appelés des mésadaptés socio-affectifs (Marineau), et la mise en place d'une aide susceptible de leur être fournie.

Une telle intégration soulève immédiatement des difficultés. Mentionnons la prise de conscience d'un double étiquetage possible : celui du professionnel qui, sans doute refuse de poser le problème en termes de délinquance, mais qui, étant donné son titre, son cadre de référence, est vu comme le représentant de ces instances définisseuses de normes et pour cette raison ségrégatives; d'autre part, celui des mineurs qu'à la suite des pratiques de sélection mises en place, à la suite de leur identification comme mésadaptés, risquent d'être repérés et catégorisés comme c délinquants ?, comme "dangereux », comme * difficiles ", etc., c'est-à-dire, comme la clientèle particulière de ce nouveau type de professionnel.

Dans cette extension du champ d'activité qui touche à la prévention, il y a donc indiscutablement un danger : celui qui consiste à * criminaliser * un cas, tout comme le psychiatre pourrait naturellement tendre à le * psychiatriser *, c'est-à-dire à le définir d'une manière telle qu'il entre dans le cadre de sa compétence. Le problème n'est pas simple, car éviter ce danger ne devrait pas devoir signifier une abstention devant des cas qui effectivement risquent d'évoluer d'une manière malheureuse, non seulement lorsque l'on prend comme critère l'adaptation sociale, mais aussi et surtout l'épanouissement personnel; néanmoins, dans la logique đu développement d'une profession, que ce soit celle de criminologue, de psychologue ou de psychiatre, il y a une tendance à définir les individus comme clients espérés ou souhaités, et, dans la mesure où les professionnels ont un pouvoir de contrainte (directe ou indirecte), d'utiliser ces contraintes pour les besoins de la cause.

Il est peut-être heureux que ce fait soit particulièrement : visible ? pour le criminologue ou pour tous les cas qui touchent à l'inadaptation sociale, car la prise de conscience d'une contrainte possible est presque incluse dans la manière dont on nomme ce professionnel et dont on définit l'acte, raison de l'intervention. Cette réalité nous explique sans doute qu'à la suite de ce long cheminement commencé sous le signe de ce que l'on a appelé la × criminologie scolaire $\triangleright$, se sont développées tellement d'initiatives dans le cadre de la prévention, ou plutôt dans le cadre que l'on préfère ne plus appeler la prévention, mais la mise en place d'un milieu adéquat à un développement personnel.

Dès lors, lorsque nous avons dit, en commençant ce paragraphe, que dans le domaine de la criminologie, au Québec, le mouvement se prouvait en marchant, ce n'était pas une figure de style. C'était un choix qui, à travers les interrogations qui se sont posées, s'est révélé particulièrement fécond.

Il est plus difficile de décrire les apports que nous trouvons dans les Acta en ce qui concerne la théorie criminologique et les travaux qui se situent dans ce cadre. Il importerait, en effet, de préciser une manière d'ordonner ces apports, et dans ce domaine, tout ordonnancement est discutable.

On pourrait sans doute utiliser le clivage à partir duquel il est devenu traditionnel de décrire l'évolution des sciences criminologiques : celui qui sépare une criminologie du passage à l'acte et une criminologie de la réaction sociale. Mais un tel clivage pose un problème dans la mesure où l'on se contente de le faire porter sur les thèmes envisagés (personnalité et acte délinquants d'une part, réaction sociale d'autre part), et non sur l'attitude du chercheur, quel que soit son objet. Ce qui, en effet, 
nous paraît constituer la contribution essentielle de la criminologie de la réaction sociale est la modification d'attitude qu'elle a introduite dans l'étude du phénomène délinquant : l'acte ou l'individu délinquants, tels qu'ils sont a atteints * par le chercheur ou même par le clinicien, ne constituent pas des entités objectives dont la nature serait d'être e délinquants ». Le fait même qu'ils soient appelés tels, résulte du fait qu'ils ont été définis socialement de cette manière, que pour l'être, ils ont fait l'objet d'un processus de sélection qui ne se déroule pas au hasard, qu'en plus, cette définition constitue ou a constitué un élément essentiel de la situation dont il est indispensable de tenir compte. En d'autres termes, après les critiques des tenants de la réaction sociale, il est difficile d'aborder la délinquance avec ce que nous pourrions appeler une conscience naĩve : les termes de délinquants et de délits nous réfèrent essentiellement à une construction sociale, et il importe d'en tenir compte, quel que soit le niveau d'analyse auquel on se situe.

Lorsque nous regardons l'ensemble des travaux qui ont paru dans les Acta, nous constatons qu'un certain nombre d'entre eux pourraient se regrouper autour du thème : personnalité, acte délinquant, que d'autres pourraient l'être d'une manière plus spécifique autour de l'étude des réactions sociales. Ce fait ne nous permet cependant pas de les situer pour autant de part et d'autre de cette ligne de partage que nous venons de préciser ; car l'attitude des auteurs du premier groupe n'apparaît nullement comme etant l'expression d'une conscience naïve "; si certaines de leurs déductions posent un problème, ils intègrent en quelque sorte cette dimension de la réaction sociale dans la construction de leur objet. Par contre, les travaux que nous situons dans le second groupe, tout en portant sur la réaction sociale, peuvent de la même manière poser un problème du fait que leur objet apparaît comme plus ou moins adéquat par rapport à des élaborations plus nuancées.

La relecture des numéros de ces dix dernières années nous laisse dès lors l'impression d'un effort poursuivi dans des orientations dont chacune acquiert sa complexité propre. $\mathrm{Si}$ nous regroupions pour la facilité ces contributions autour des thèmes d'une part de la personnalité et de l'acte délinquant, et d'autre part de la réaction sociale, cette division est relativement arbitraire, et le fait de le constater ne manque pas d'intérêt.

Autour d'une problématique axée sur une prise en considération de la personnalité đélinquante ou déviante, nous avons principalement les travaux de M. Fréchette (1970) et de Fr. Goyer-Michaud (1973), mais aussi ceux d'E. Douyon (1969) et de M.A. Bertrand (1969).

Les études de Fréchette et de Goyer-Michaud nous fournissent des résultats précis sur des caractéristiques que nous retrouvons chez, les individus délinquants lorsqu'on les compare à des non-délinquants ou lorsqu'on opère dans le groupe même des délinquants des distinctions selon le degré de récidive. Fréchette dégage à partir des données fournies au moyen de l'épreuve de Kelly le fait que les délinquants, et principalement les délinquants récidivistes, manifestent ce qu'il appelle un sentiment d'éloignement interpersonnel, c'est-à-dire une capacité moindre de recevoir l'autre ou de s'identifier à lui. Pour sa part et en se référant plus particulièrement aux jeunes, Goyer-Michaud, dans le cadre d'une étude sur les valeurs, isole ce qu'elle appelle les valeurs motivantes, c'est-à-dire les valeurs directement engendrées par l'anxiété qui, selon elle, paraît bien être à l'origine de tout comportement moral. Ces valeurs motivantes peuvent s'organiser 
différemment selon le type d'anxiété qui les provoque : d'après l'hypothèse de Goyer-Michaud, en comparant les délinquants à leurs pairs socialement adaptés, les premiers seraient plus sensibles à l'anxiété hédonique (c'est-à-dire une anxiété dominée par la crainte d'un déplaisir) et à l'anxiété allocentrique (c'est-à-dire au malaise résultant d'une perte d'estime auprès des pairs), de telle sorte que leurs comportements seraient déterminés par ces valeurs motivantes particulières; par contre, chez les pairs non délinquants, seraient plus nettement créatrices du cadre de valeurs l'anxiété d'autorité (c'est-à-dire la peur de provoquer la réprobation des différentes figures d'autorité) et l'anxiété d'intégrité (liée à l'estime de soi).

Sans qu'on ne puisse évidemment dire que ces perspectives se superposent, l'une et l'autre néanmoins soulignent une réalité que Fréchette a particulièrement bien mise en lumière : la criminalité ou la délinquance ne s'expriment pas uniquement en termes de conduite, mais aussi en termes de structure mentale. C'est à ce niveau que se pose un problème majeur : celui de la personnalité criminelle, ou en d'autres termes, celui d'une * structure actuelle de personnalité, qui, en cas de comportement récidivant, paraît bien être l'élément dominant qu'il importerait de prendre en considération, tandis que la délinquance épisodique s'expliquerait beaucoup plus par l'expérience sociale vécue dans le présent, sans que les caractéristiques de personnalité ne soient particulièrement en cause. C'est ainsi que pourrait se résumer l'hypothèse de départ, reposant sur ces deux niveaux d'explication de la délinquance. Dans la terminologie systémique utilisée par $M$. Le Blanc, on maintiendra qu'un système de forces de nature sociologique pourrait opérer avec d'autres systèmes de forces (de nature psychologique ou biologique), l'un et l'autre de ces systèmes pouvant être prépondérants selon les cas $(1972,1974)$.

Le problème consiste à se demander si une hypothèse de cet ordre n'est pas surtout satisfaisante au niveau général d'une explication du passage à l'acte, le fait de se situer à ce niveau ayant naturellement pour conséquence de voiler d'autres éléments qui pourtant seraient essentiels. On constate en effet que dans son étude de 1970, Fréchette a clairement souligné que cette capacité d'établir des contacts avec des autruis signifiants (et qui apparaît moindre chez les délinquants que chez les nondélinquants), est sérieusement altérée par la situation d'emprisonnement : cette capacité est d'autant moins présente que le nombre d'années passées en prison est plus élevé. D'autre part, il constate aussi que la distanciation par rapport aux figures parentales, dans certaines catégories de délinquants envisagés, est compensée par l'établissement d'un lien de plus en plus affirmé avec les pairs délinquants, ce qui indiscutablement oriente le sujet dans la voie d'une délinquance assumée.

$\mathrm{Si}$ nous soulignons l'intérêt de ces constatations, c'est parce qu'elles nous font entrer dans ce que nous pourrions appeler la logique même selon laquelle une personnalité se modifie pour acquérir cette structure mentale actuelle * qui paraît d'une importance prépondérante dans l'explication du passage à l'acte. Néanmoins, faut-il pour autant mettre l'accent sur cette estructure actuelle, et sur la nécessité d'élaborer un outil de diagnostic qui soit le plus nuancé possible afin de pouvoir repérer ceux chez lesquels cette structure existe et ceux chez lesquels elle n'existe pas. On peut se demander s'il n'en résulte pas automatiquement une élimination de ces processus du champ d'attention du clinicien comme du chercheur, une réduction des divers processus possible à ce qui paraît être leur résultat au niveau des traits de personnalité. Il me paraît de plus en plus 
nécessaire de choisir l'une ou l'autre de ces optiques et que vouloir les prendre simultanément est contradictoire. Cela ne veut pas dire que l'une soit fausse et que l'autre soit vraie, que l'une soit utile et que l'autre ne le soit pas. Nous dirions plus simplement que l'une (l'optique axée sur le passage à l'acte), en prenant comme point de départ la notion de personnalité actuelle, voile ou réduit trop d'éléments qui paraissent essentiels, car c'est, semble-t-il, au niveau de la prise en considération de ces éléments que se situent les véritables possibilités de compréhension et d'action.

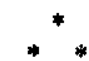

D'une manière sans doute paradoxale, nous pourrions trouver dans les articles d'E. Douyon et de M.A. Bertrand quelques-unes de ces dimensions éliminées.

Le premier paraît traiter un sujet d'ethnologie qui, à première vue, n'a pas grand-chose à voir avec la délinquance : la transe vaudouesque : un symptôme de déviance psychoculturelle. Sans doute, le lecteur pourrait-il devenir moins catégorique lorsque sous la plume de l'auteur, il lit que l'existence de ce système magico-religieux de la transe explique peut-être, en Haïti, le nombre peu élevé de délinquants et de suicidés.

$\AA$ propos de cette recherche d'E. Douyon, nous ne voudrions insister que sur un seul point : la personnalité des a criseuses ,, c'est-à-dire des personnes qui tombent en transe et sont comme possédées, révèle une dimension anxieuse et dépressive plus nette que celles du groupe contrôlé. Cependant, l'analyse nous montre que le problème ne se pose pas uniquement, ni même essentiellement, au niveau du cas individuel ou des particularités individuelles. Sans doute, la transe paraît bien être une alternative par laquelle le sujet - un sujet probablement plus vulnérable que les autres - trouve une porte de sortie à cette situation anxieuse et d'asphyxie qu'il connaît et que connaît son groupe social (une analyse sur un phénomène du même ordre, le tarentisme, a été menée en Italie du Sud par E. de Martino, 1961). Mais il importe d'insister sur le fait que cette alternative, ou cette < solution s est en quelque sorte fournie par le groupe. Elle constitue une élaboration culturelle qui non seulement donne à ces sujets une porte de sortie socialement acceptée mais permet à l'ensemble du groupe de participer et de vivre, à travers l'expérience extatique de l'un de ses membres, un déblocage de cette tension due aux conditions mêmes dans lesquelles il vit. Et en cela, ce fait constitue effectivement une voie de canalisation des impulsions.

Peut-on dire que ces constatations sont, d'une manière ou d'une autre, transférables à notre propre culture ? Sans doute, une solution magique de ce genre repose sur une situation d'impuissance, ou de fatalité vécue comme inéluctable, à laquelle le groupe social se trouve confronté. Dans le cadre d'une civilisation de type prométhéen, de telles solutions collectives deviennent de moins en moins possibles, parce que d'autres voies sont ouvertes, toutes plus ou moins axées sur la valorisation d'une prise en charge rationnelle de son destin. Ce pourrait être, de la part du groupe lui-même, une prise en charge du destin collectif, avec tout ce que cela pourrait impliquer. Là où le système valorise plus nettement, comme solution, la prise en charge individuelle, la délinquance, c'est-à-dire le nonrespect des moyens préconisés par le groupe pour le faire, apparaît nécessairement et d'une manière plus généralisée comme réponse possible aux limitations imposées. En d'autres termes, face à ce que certains peuvent 
vivre comme fatalité, aucune issue, ou très peu d'issues (le jeu de hasard? la drogue ?) sont offertes qui se situeraient au niveau magique ». Tout se passe d'ailleurs comme si cette fatalité, qu'un moment on a cru pouvoir évacuer sans trop de problèmes, n'était pratiquement pas évacuable dans un tel type de société, et que même dans toute société, il existait un certain nombre de réalités inéluctables, dont particulièrement la mort, qu'il était particulièrement difficile de prendre rationnellement en charge. Quel rôle joue, dans un tel système, le délinquant $?$ Celui de bouc émissaire? Celui d'inavouable objet d'identification? Celui d'initiateur? Tout dépend évidemment du type de délinquance dont il s'agit. La personnalité de ces sujets peut fort bien présenter certaines caractéristiques, comme celles de ces criseuses; mais ces caractéristiques ne nous renvoient-elles pas justement aux données constitutives du système? Ce sont là des questions qui sont recouvertes par une théorie de la personnalité criminelle et que l'on ne peut atteindre que par l'étude et l'interprétation des processus plus particuliers auxquels nous avons fait allusion plus haut.

D'autre part, comment poser le problème de la réaction de la société à la délinquance ? $M$. Cusson (1974) a raison d'affirmer que devant le manque de cohérence des systèmes répressifs actuels, il est impossible d'avoir une théorie cohérente de la peine. Son effort pour chercher à resituer celle-ci dans une logique qui ne se limiterait pas au cas individuel, mais qui s'intégrerait dans une nouvelle logique sociale (1976) nous situe au niveau où de nouvelles solutions sont possibles.

Un problème du même ordre se pose dans la recherche de M.A. Bertrand (1969). Sans doute, celle-ci met-elle surtout l'accent sur la réaction sociale à la délinquance à partir d'une représentation de la criminalité masculine et féminine. Mais en débouchant, à propos des femmes, sur la notion de rôle, et de rôle intériorisé, l'auteur découvre un des éléments constitutifs de la personnalité, ou en tout cas, un élément que l'on pourrait considérer comme indissociable des $<$ traits $>$ de personnalité, tout particulièrement dans une perspective comme celle d'Erikson axée sur la problématique : perception de soi comme a agent " ou comme * objet *. On aimerait sans doute avoir des informations plus précises sur certaines variables, mais il se dégage de l'étude que si la femme délinquante (qui se perçoit comme objet ou comme victime) présente des caractéristiques traduisibles en termes de personnalité, il est cependant nettement préférable d'envisager celles-ci à travers le concept de rôle qui situe la personne dans le cadre d'une interrelation et dans celui d'une structure sociale qui, dans ce cas, est effectivement déterminante Mais encore faut-il, néanmoins, que le concept de rôle soit pris dans un sens suffisamment complexe : il ne nous réfère pas uniquement aux statuts sociaux dont les rôles seraient l'expression, mais aussi à la manière dont le sujet (en l'occurrence la femme) peut se définir par rapport à ses désirs, par rapport aux désirs des autres et au jeu subtil des interdictions qui les règlent.

Nous pourrions résumer le débat de la manière suivante : confrontée à la notion de personnalité délinquante, ou de structure actuelle de personnalité, notre attitude reste ambivalente. Cette notion correspond effectivement à une réalité clinique qu'il est utile de dégager et qui donne au juge et au clinicien un outil lui permettant de distinguer des autres une personnalité structurée dans le sens d'une délinquance assumée et persistante, du moins, pour autant qu'il s'agisse d'un certain type de délinquance. Mais 
l'optique prise n'a-telle pas pour conséquence d'évacuer les divers processus susceptibles de modifier la personnalité ? D'évacuer également les structures sociales et les schémas culturels déterminant à la fois les rôles, les possibilités de satisfaction, les canaux selon lesquels celles-ci doivent et peuvent se faire ? C'est-à-dire, d'éliminer des éléments qui nous paraissent déterminants et, également, les plus susceptibles d'être atteints par une action ou une intervention. Il nous est évidemment impossible de prolonger ce débat. On pourra néanmoins constater que les Acta comme d'ailleurs les diverses recherches poursuivies à l'École de criminologie nous fournissent les éléments nécessaires pour le poser dans toute son ampleur, mais aussi, dans sa complexité.

Des monographies sur des catégories d'actes délinquants peuvent à ce point de vue être extrêmement utiles. A. Normandeau (1972) nous donne a ce propos une étude des vols qualifiés et de leur rapport avec la violence. L'avantage de telles études appelées phénoménologiques au sens où elles décrivent un phénomène, est de porter sur une conduite, en tenant compte des divers éléments constitutifs de ce qu'est une conduite. Les caractéristiques qui ont trait à l'auteur de l'acte sont sans doute très réduites, mais celui-ci n'est défini que comme un des éléments de la situation qui, selon le type d'acte envisagé, peut présenter des particularités qu'il est essentiel de connâ̂tre. De telles études nous fournissent, dès lors, une toile de fond susceptible de donner à l'analyse de cas individuels leur véritable signification. A ce niveau également, nous devons reconnaître que la théorie de la personnalité criminelle, du fait que les mêmes caractéristiques paraissaient se retrouver quel que soit l'acte commis, a eu pour conséquence d'amener le criminologue clinicien à négliger les particularités propres à chaque type d'acte considéré comme conduite, c'est-à-dire, comme lié à un ensemble particulier de données situationnelles. Là aussi nous parlerons d'un certain * aplatissement $\star$ des différences.

Les travaux que nous pourrions regrouper autour d'une prise en considération de la réaction sociale abordent ce thème sous des angles différents. Nous essayerons de les expliciter brièvement, sans entrer cependant dans un débat comme nous l'avons fait pour la partie précédente, car, ayant une formation de clinicien, nos positions s'affirment naturellement d'une manière moins nette.

Le premier thème développé par $D$. Szabo dans un article qui, en 1968, introduisit d'ailleurs la collection des Acta, est celui d'une explication de la délinquance juvénile à partir d'un conflit de valeurs entre générations, les adultes ayant spontanément une attitude misotrope, c'est-à-dire conservatrice des valeurs en place, alors que celle de la jeune génération sera plus particulièrement néotrope, c'est-à-dire, visera à introduire une nouvelle manière d'être et de vivre. Ce clivage entre générations tendrait à remplacer le clivage résultant des classes sociales et développe une réaction qui vise à définir comme délinquants ou déviants des actes que les adolescents perçoivent comme correspondant à leur culture propre. Le problème est donc nettement posé en termes de conflit de cultures et l'accent sera mis sur l'anomic au sens durkheimien du terme, c'est-d-dire comme perte de solidarité.

Cette perspective a été présentée à différents niveaux : au niveau macro-sociologique par D. Szabo et dans la suite, par Szabo et Gagné (1972) ; au niveau d'un instrument pour mesurer les attitudes morales et vérifier l'hypothèse đe départ, par les collaborateurs de Szabo (M. Le Blanc, 
L. Deslauriers et D. Gagné, 1968), par la suite, comme nous l'avons déjà signale, par F. Goyer-Michaud (1973) et finalement, au niveau d'une analyse comparée des systèmes juridiques mis en place pour répondre ou, dans une perspective plus progressiste, selon les vœux de l'auteur, pour * assumer * la délinquance juvénile, par A. Parizeau (1972).

L'ensemble de ces contributions exprime manifestement une nouvelle manière de poser le problème de la délinquance juvénile en rapport avec un nouveau type de société qui, à l'époque, paraissait se développer irrésistiblement. Nous utilisons l'imparfait, car cette perspective à l'heure actuelle est moins nette, en ce sens qu'elle repose sur l'hypothèse que, dans ces sociêtés post-industrielles, une croissance économique jugée illimitée justifie la croyance d'un dépassement des clivages sociaux tout en créant, comme le souligne d'ailleurs Gagné, un nouveau type d'aliénation qui sera celle du consommateur. Cette perspective n'est plus que partiellement vraie. La croissance économique se trouve freiné par des facteurs non seulement peu contrôlables, mais dont le rôle de frein risque d'aller en s'accentuant, et on ne peut plus dire que les clivages sociaux tendent à s'estomper de la manière dont il était prévu. De même et parallèlement, l'attitude des jeunes tend à se caractériser plus nettement par une attitude de repli dans ce que certains sociologues ont appelé des niches ou des refuges écologiques. Il y aurait là un débat qu'il serait extrêmement intéressant de poursuivie.

Un deuxième thème, devenu depuis lors plus classique, est celui de la mesure de la criminalité en tenant compte d'une donnée qui est habituellement négligée dans les statistiques officielles : la gravité relative de l'infraction. Il est certain que l'indice de Sellin et Wolfgang constitue à ce propos un progrès considérable dans la manière de traduire, pour un pays ou une région donnés, la réalité criminelle. D. Akman et A. Normandeau ont présenté, dans leur article de 1968, une réplication de cette méthode pour le Canada. Un tel indice, comme nous pouvons l'imaginer, repose sur l'appréciation, faite par un échantillon de sujets choisis par le chercheur, de la gravité relative d'un certain nombre d'infractions.

Une de ces critiques soulevées par la suite porte sur les caractéristiques mêmes de cet échantillon : peut-on se contenter, pour élaborer l'indice de gravité, d'un échantillon pris globalement, celui-ci fut-il même représentatif de la population, ou faut-il considérer, dès le départ, qu'il existe dans cette population des divergences suffisamment importantes, liées par exemple aux classes sociales, pour qu'il faille éviter que celles-ci ne soient voilées par ce procédé ? En d'autres termes, cette méthode repose sur l'hypothèse d'un consensus de la population à propos des jugements portés. L'hypothèse ne doit-elle pas être que le dyssensus constitue la réalité de base (v. P. Robert et collab., 1976) ? Une telle optique aurait pour conséquence de favoriser des études portant sur des échantillons diversifies, et surtout, d'approfondir une perspective plus qualitative, non seulement dans ce domaine spécifique, mais dans celui, plus large (et ce serait là notre troisième thème), de l'image que l'on a de la justice pénale. Cette analyse, plus qualitative, aboutit d'ailleurs à poser le problème en termes non plus d'opinion, mais d'attitude, ce qui soulève effectivement d'autres questions.

Les enquêtes menées sur le fonctionnement de la justice furent, comme nous l'avons dit, les premières grandes recherches du département dans le cadre des commissions officielles (voir supra). Dans ce domaine et à un niveau plus vaste, de nouveaux projets d'enquête viennent d'être mis 
sur pied conjointement par le C.I.C.C. (D. Szabo et Y. Brillon) et par l'École de criminologie (A. Normandeau). On peut se demander, à la suite de l'apport des travaux de P. Robert et de C. Faugeron, dans quel sens se poursuivra l'analyse. L'intérêt d'une approche plus qualitative dans ce domaine réside dans le fait que la répartition des opinions, des jugements, et même des attitudes ne prend de véritable sens que dans la mesure où on peut les resituer dans les représentations mentales ou dans l'univers mental de ceux qui les émettent ou les manifestent. Il importe, dès lors, comme l'écrit C. Faugeron (1975), de recueillir le \& discours》 de ceux auxquels on demande de donner un avis sur le fonctionnement de la justice, et non plus de simples réponses; c'est à ce moment que les méthodes qualitatives prennent leur importance. Si nous en parlons, c'est qu'effectivement, dans le cadre même de l'École, et sous l'impulsion de G. Tardif, cette dimension est explicitement abordée, dans un tel ordre de préoccupation, à côté et parallèlement aux méthodes quantitatives.

Nous n'en avions pas fini de parler de la mesure de la criminalité, notre deuxième thème, car à côté d'un affinement lié au fait de prendre en ligne de compte la gravité relative de l'infraction, un autre affinement consiste à introduire la délinquance cachée dans la représentation d'ensemble que l'on a des comportements délinquants, ou plus exactement, à vouloir atteindre la délinquance * réelle *.

Nous aurons à suivre ici les diverses recherches de $\mathbf{M}$. Le Blanc qui, au niveau de la délinquance juvénile, a utilisé successivement comme méthode de reperage les informations fournies par la police (1968) et d'autre part la délinquance révélée par la passation du test de Nye et Short, dans ce dernier cas, tout particulièrement, en vue de mesurer l'impact de la classe sociale sur la délinquance (1969). Alors que la première étude révèle clairement que dans les divers secteurs de recensement, le volume de la délinquance diminue à mesure que le statut social s'améliore, la deuxième au contraire indique que, d'une manière globale, il n'existe pas de différence entre les deux échantillons définis à partir de la classe sociale, mais que des différences apparaissent au niveau de certains types de comportements qui tantôt seront plus fréquents dans la classe aisée (fugue, défi aux parents, utilisation de drogue, etc.), tantôt dans la classe ouvrière (batailles). A partir de ces indications de base, Le Blanc a mené deux recherches publiées dans les Acta : l'étude détaillée d'un comportement caractéristique des milieux aisés, une observation participante d'un groupe d'adeptes de la drogue (1972), et d'autre part, une analyse de la réaction sociale à la délinquance juvénile (1971), analyse rendue en effet nécessaire par cette contradiction que nous avons soulignée : sur l'échelle de la délinquance révélée, il y a un pourcentage à peu près égal de délinquants dans un quartier ouvrier et dans un quartier aisé alors que par contre, il y a deux fois plus de délinquants officiels dans le même quartier ouvrier que dans le quartier aisé. Pour analyser les mécanismes en jeu, l'auteur utilise d'une manière judicieuse la technique de prédiction par attributs dichotomiques. Il nous est impossible de décrire les résultats en détail. Nous dirons simplement que si le milieu socio-économique influence nettement l'entrée dans le système de la justice juvénile ainsi que la réaction judiciaire, ce sont par contre les caractéristiques des événements délinquants qui déterminent les sanctions.

Le dernier thème qu'il reste encore à aborder est celui de la mesure pénale qui a fait l'objet de diverses contributions. Nous avons déjà souligné la démarche de $\mathbf{M}$. Cusson dominée par son souci de donner aux pratiques sociales telles que rendre la justice, leur sens par rapport aux structures 
sociales et aux exigences qui en découlent. Dans son article de 1974 sur la peine, il critique à juste titre le caractère non seulement aléatoire, mais contraignant et souvent stigmatisant du traitement, ce terme laissant croire que la vie thérapeutique constitue l'élément essentiel de ce genre de mesure. Effectivement, il serait préférable de nommer les choses par leur nom et de reconnaître le caractère correctif de tout traitement envisagé dans ce cadre. Cela veut-il néanmoins dire que la réaction sociale à un acte désapprouvé (nous préférerions dire : à un acte qui pose problème au groupe) doive nécessairement être une peine ? Le procès (pénal ?) dans une certaine mesure ne pourrait-il pas devenir une négociation entre plusieurs instances, la société, la victime, le coupable, dont chacun aurait un certain point de vue? Les derniers commentaires de Cusson (1976) à propos du concept d'échange, laissent croire en une telle évolution. Il faudrait aussi, à son propos, mentionner les recherches, faites par lui et son équipe dont Mme Laflamme-Cusson, sur les mesures de rééducation des mineurs et la manière d'en évaluer la portée. Mais celles-ci ne se sont pas traduites par une contribution aux Acta.

Finalement, il convenait que le point final de cette chronique portant sur huit années des Acta criminologica soit, à propos de ce quatrième thème, le rappel, d'une part, des droits de la victime. Il a toujours existé, au sein du département de criminologie, un intérêt considérable pour tout ce qui touchait à la victimologie. H. Ellenberger et E. Fattah ont brillamment représenté ce courant. Dans son article de 1968, J. Rico pose le problème en d'autres termes : la victime n'est pas seulement l'éventuel participant d'une interrelation dont le dénouement a pu être tragique. C'est également un être dont certains droits ont été lésés. Il importe que toute justice pénale puisse en tenir compte, car dans l'une comme dans l'autre optique, la victime est bien souvent la grande absente du procès pénal tel qu'il se déroule actuellement.

D'autre part, il importe également de rappeler les droits de l'homme privé de liberté. Dans sa contribution sur l'application des règles minima pour le traitement des détenus, P. Landreville (1973) constate que ces règles, définies par le premier Congrès des Nations-Unies pour la prévention du crime et le traitement des délinquants, ne sont pas protégées par des lois et que dans les institutions fédérales ou provinciales, elles ne font l'objet d'aucun contrôle et que n'est prévu aucun système de recours. Et encore, on peut dire que ces règles minima ne portent que sur les droits essentiels et qu'il faudrait en arriver à ce que les droits des détenus se rapprochent le plus possible des droits du c citoyen ordinaire s. Un tel problème se pose dans la plupart des pays. On peut néanmoins se demander si les progrès décisifs, dans ce domaine, ne doivent pas nécessairement aller de pair avec une nouvelle manière de concevoir la justice. Et il faut bien reconnaître que la nouvelle loi sur les délinquants dangereux ne va pas précisément dans ce sens.

Tout en essayant d'être, dans cette chronique, le plus attentif possible à toutes les contributions, et même, aux prolongements que celles-ci peuvent avoir eus dans certains séminaires ou publications polycopiées, il est évident que nous avons nécessairement dâ laisser dans l'ombre certaines activités dans la mesure où elles ne se sont pas exprimées à travers les Acta. Il faut bien reconnaître que celles-ci se déploient dans tellement 
de directions qu'il est pratiquement impossible de les rassembler dans un texte aussi bref.

De même, on aura pu remarquer que nous n'avons fait aucune allusion aux articles parus dans la revue publiée sous sa nouvelle forme. C'est qu'en réalité, celle-ci traite, dans chacun de ses numéros, un thème particulier. Chacun de ces numéros exigerait, dès lors, une discussion centrée sur le thème envisagé, ce qui nous aurait engagé dans une nouvelle tâche que l'on pourrait, peut-être, réserver pour plus tard. Cette chronique constitue dès lors une sorte de prolégomène à la lecture de la revue, nouvelle forme, puisqu'elle dresse l'état de la question avant que les Acta ne se soient modifiés.

Une troisième partie aurait été nécessaire, que nous ne présenterons que d'une manière symbolique parce qu'elle exigerait un développement considérable qui, cette fois, ne nous situerait plus uniquement dans le cadre québécois, mais dans le cadre international. C'est celle qu'il importerait de consacrer aux activités des centres, et principalement, aux activités du C.I.C.C. menées sous la direction de D. Szabo et du secrétaire général A. Parizeau. Les Acta ont rendu périodiquement compte de ces activités (1970, 1971, 1973, 1974), ou plus exactement, des symposia internationaux qui constituent une des formes que celles-ci ont prises.

Si nous avions à distinguer les différents secteurs de préoccupations, nous retiendrions d'une part les problèmes généraux qui se posent dans les pays industriels ou postindustriels à propos d'une étude comparative, non seulement de la criminalité, mais aussi de tout ce qui touche à l'organisation de la justice. Face à ce dernier problème, un point de vue paraît avoir été privilégié : celui qui consiste à le poser en termes de coûts et bénéfices. Nous aurions, d'autre part, le secteur de la criminologie clinique comparée, qui s'est concrétisée dans l'organisation de journées internationales auxquelles ont collaboré l'Institut Pinel et le Centre de criminologie clinique de Gênes et dont l'ensemble des textes constitue une mine extrêmement riche d'informations. Et finalement, le C.I.C.C. a également poursuivi dans les pays en voie de développement rapide (et principalement en Afrique) des analyses transculturelles et dans ce cadre, une investigation de la manière particulière dont se pose le problème de la délinquance et de l'administration de la justice. Nous devrons principalement citer les comptes rendus de S. Arcand et Y. Brillon (1973) ainsi que de S. Rizkalla (1974).

Il va de soi que chacun de ces secteurs constitue en lui-même un univers tellement vaste que nous devons effectivement nous contenter de les évoquer d'une manière symbolique. 